\title{
Chemical Versus Thermal Folding of Graphene Edges
}

\author{
Ninghai Su, Miao Liu, and Feng Liu ( $ه)$ \\ Department of Materials Science and Engineering, University of Utah, Salt Lake City, UT 84112, USA \\ Received: 14 August 2011 / Revised: 26 September 2011 / Accepted: 27 September 2011 \\ (C) Tsinghua University Press and Springer-Verlag Berlin Heidelberg 2011
}

\begin{abstract}
Using molecular dynamics (MD) simulations, we have investigated the kinetics of the graphene edge folding process. The lower limit of the energy barrier is found to be $\sim 380 \mathrm{meV} / \AA \AA$ (or about $800 \mathrm{meV}$ per edge atom) and $\sim 50 \mathrm{meV} / \AA$ (or about $120 \mathrm{meV}$ per edge atom) for folding the edges of intrinsic clean single-layer graphene (SLG) and double-layer graphene (DLG), respectively. However, the edge folding barriers can be substantially reduced by imbalanced chemical adsorption, such as of $\mathrm{H}$ atoms, on the two sides of graphene along the edges. Our studies indicate that thermal folding is not feasible at room temperature (RT) for clean SLG and DLG edges and is feasible at high temperature only for DLG edges, whereas chemical folding (with adsorbates) of both SLG and DLG edges can be spontaneous at RT. These findings suggest that the folded edge structures of suspended graphene observed in some experiments are possibly due to the presence of adsorbates at the edges.
\end{abstract}

\section{KEYWORDS}

Adsorption, graphene, kinetics, molecular dynamics

Graphene has attracted much recent interest due to its fascinating physical and chemical properties and its potential applications in future nanoelectronics [1] One fundamental question for graphene is its thermal and chemical stability, since this has important implications for its practical applications. As an ultrathin two-dimensional structure only one-atomiclayer thick, graphene can undergo large out-of-plane thermal fluctuations [2]. The presence of free edges makes the graphene susceptible to edge defects and chemical impurities [3] as well as to mechanical edge warping and twisting instability [4-6]. Furthermore, several recent experiments [7-10] have observed folded graphene edges, which raises several interesting questions: (1) Whether the "folded edges" are an intrinsic or extrinsic property of graphene edge stability? (2) What is the kinetic energy barrier associated with the folding process? (3) How does the folding process depend on the chemical environment? Our aim here is to answer these fundamental questions by comparing the thermal and chemical folding processes at graphene edges, using molecular simulations. It is worth pointing out that understanding the graphene edge folding process is not only of scientific interest, but also of technological importance. For example, one very recent experiment has shown that the transport properties of open and closed graphene edges are very different, with the closed edge exhibiting a much higher conductivity [11].

A suspended free-standing graphene edge has been observed by transmission electron microscopy (TEM) to fold over [7, 8], forming a structure similar to one-half of a single-walled carbon nanotube (SWCNT). Using high resolution TEM, Liu et al. [9] have further

Address correspondence to fliu@eng.utah.edu 
shown that both armchair and zigzag edges of graphene exhibit a folded edge state. Moreover, in situ observation of multi-layer graphene has demonstrated that both the armchair and zigzag edges can form folded structures spontaneously at about $2400 \mathrm{~K}$ [10]. On the other hand, a theoretical study using a coarsegrained hierarchical multiscale model has shown that the folded edges are thermodynamically stable beyond a critical folded length [12], and a kinetic Monte Carlo calculation was consistent with the observed spontaneous folding process at high temperature ( 2400 K) [10]. Recently, quantum molecular dynamics simulations also uncovered the roles of edge reconstruction, carbon vacancies and interstitials in the thermal folding process of double-layer graphene (DLG) at high temperature (1000 K), which explained why the closed edge structure forms under Joule heating only if the sample is not irradiated by highenergy electrons [13]. Another simulation studied folding of a Y-shape graphene ribbon at high temperature [14].

Although previous theoretical studies [10,12,13] have confirmed folding of single-layer graphene (SLG) and DLG edges at high temperatures, the kinetics associated with the graphene edge folding process, which determines the feasibility of edge folding at different temperatures (including room temperature) and in different chemical environments, remains unexplored. Most importantly, the energy barriers associated with the folding processes of both SLG and DLG edges remain unknown. We noticed that the graphene preparation methods used in Refs. [7-9] all involved initially working in solution without subsequent purification steps. For example, the graphene membranes prepared by Meyer et al. [7] were treated in water, isopropanol, acetone and liquid carbon dioxide, whilst Liu et al. [9] used heat treatment of graphite powder dispersed in ethanol by ultrasonication before dropping the resulting graphene onto a grid. Thus, these graphene membranes may be "polluted" by adsorbates, which may chemically assist the graphene edge folding processes [14]. Therefore, besides "thermal folding" of clean graphene edges, i.e., a thermally activated process, "chemical folding" of graphene edges with adsorbates, i.e., a chemisorptionassisted process should be considered. In particular, we must determine the energy barriers for thermal versus chemical folding of graphene edges, to assess the feasibility of graphene edge folding processes at different temperatures and in different chemical environments.

We have carried out extensive molecular dynamics simulations to derive the limiting kinetic energy barriers for edge folding processes of both SLG and DLG, using a modified form of the bond-order potential due to Brenner et al. [15] and a similar setup as before [14]. Our own Lennard-Jones (L-J) potential parameters [14] were developed to give a $\sim 42 \mathrm{meV} /$ atom interlayer cohesive energy for graphite, in accordance with experiment [16]. We studied $\mathrm{H}$ adsorption as a simple model system for chemical folding, using a new bondorder term for $\mathrm{C}-\mathrm{H}$ to give a $\mathrm{H}$ adsorption energy of $\sim 0.9 \mathrm{eV}$ on graphite, as predicted by first-principles calculations [14]. (The original Brenner potential predicted a $\mathrm{H}$ adsorption energy about $1.7 \mathrm{eV}$ higher). For all the molecular dynamics (MD) simulations, periodic boundary conditions were only employed in the direction along the edge. The simulations were performed at $T=150 \mathrm{~K}$ in order to suppress thermal fluctuations. We note that because the edge folding energy barrier comes mainly from bending energy, involving no chemical bond breaking/formation energy, it can be well described by a classical potential. Thus, we opted to use a classical potential instead of a first-principles method to derive the folding energy barrier in order to save computational time. In the unfolding process, there will be bond breaking associated with van der Waals interlayer interactions. So one may expect our calculated unfolding barriers to be less accurate than the calculated folding barriers, but this will not affect our conclusions on edge folding feasibility which is solely determined by the folding barriers.

Before exploring the kinetics associated with the graphene edge folding process, we first study the thermodynamic driving force for edge folding because the folded edge structure can be more stable than the flat edges. Figure 1 illustrates the energy minimization simulations to show the stability of the folded graphene edge. We generate an edge-folded SLG sheet with an overlapping length $l$ and folding length $L$, as shown in Fig. 1(a). Then, we release the folded edge by moving the upper portion toward right along the $+x$-direction 
to reduce $l$ in steps of $1 \AA$ (from Fig. 1(a1) to Fig. 1(a3)), and the potential energy is calculated at each step, as shown in Fig. 1(b). The folded edge energy per edge length relative to the unfolded edge is

$$
E_{\mathrm{e}}=\gamma_{\mathrm{i}} \times 1+\gamma_{\mathrm{b}}
$$

where $\gamma_{\mathrm{i}}$ and $\gamma_{\mathrm{b}}$ are the interlayer van der Waals (vdW) bonding energy of the overlapping portion and the bending energy of the folding portion, respectively. $\gamma_{b}$ is a constant because the shape of the folding portion remains the same with almost constant $L=22 \AA$, as $l$ is reduced (see Figs. 1(a1)-1(a3)). In calculating the potential energy as a function of $l$ in Fig. 1(b), the energy of the flat SLG is set as the zero point of the energy (shown in Fig. 1(b) as a black dashed line).

The data show a nice linear relation as expected, and the interlayer bonding energy obtained from the linear fitting (the slope of the red solid line in Fig. 1(b)) gives $\gamma_{\mathrm{i}} \approx 15 \mathrm{meV} / \AA^{2}$, or about $42 \mathrm{meV} /$ atom, consistent with the L-J potential used. The $E_{\mathrm{e}}$ value at $l=0$ gives the bending energy $\gamma_{b} \approx 306 \mathrm{meV} / \AA$, or about $625 \mathrm{meV} /$ atom. By extrapolation, the folded edge of the SLG is seen to be more stable than the flat edge if $l>20 \AA$, when the energy gain from the interlayer vdW bonding of the overlapping portion overcomes the energy cost from the bending of the folding portion. In addition, the potential energy is microscopically

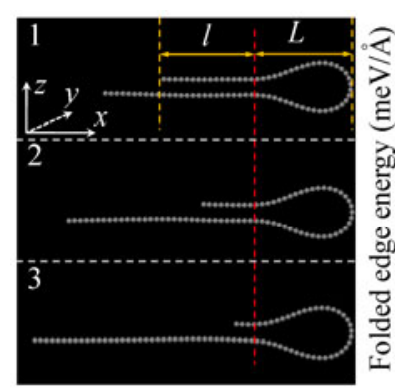

(a)

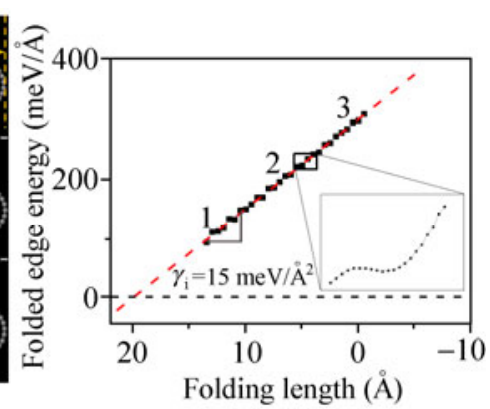

(b)
Figure 1 Energy minimization simulation of the edge-folded SLG. (a) Structures of the edge-folded SLG with different lengths of overlap portion. $L$ and $l$ are overlapping length and folding length, respectively. The grey balls are carbon atoms. The red dashed line represents the reference plane for measuring $l$ and $L$. (b) The folded edge energy as a function of folding length. Dotted line: energy of flat graphene (set to be zero). Solid line: linear fit to the data (solid black squares). The inset shows the fine structure of the $E-l$ curve affected by the atomic stacking of the overlapping portion, which leads to a periodic fluctuation of the $E_{\mathrm{e}}-l$ curve, as shown in the inset of Fig. 1(b).

Even though the folded edge structure can be thermodynamically more stable than the flat edge, the folding process may still be prohibited by a large kinetic barrier, especially for a thermally activated process. Next, we derive the energy barriers for the edge folding processes. We note that we do not attempt to directly simulate the folding process of a graphene edge, because the process is too complex and requires much too long a simulation time. So, instead, we will use MD simulation as an effective method to derive the "limiting" kinetic barriers for edge folding. We consider the folding starts locally at one place then spreads around the whole edge (like a zipping effect). The initial local folding is thus the rate-limiting step and the barrier, which should be defined by energy per length, largely determines the overall folding probability because the remaining edges have much smaller barriers per length. However, it is very difficult (if not impossible) to determine this rate-limiting barrier because it may not have a unique value, but rather depend on how the folding process actually occurs and change with edge length. On the other hand, we can establish the lower-bound of this barrier by assuming the edge folds collectively, or in other words, as soon as the initial folding starts somewhere, the remaining edges follow immediately, as we have done here.

For convenience, the unfolding process of SLG, which is the reverse process of folding, is simulated in order to obtain the energy barrier of folding as shown in Fig. 2. First, the atomic positions in a folded SLG edge are relaxed for 30 ps (Fig. 2(a1)). Then, a horizontal force is applied (the force is applied every 30 ps and sustained for $0.2 \mathrm{ps}$ periodically) on all the carbon atoms in the upper portion (both the overlapping and folding portions) of the folded edge along the $+x$-direction. The long simulation time of $\sim 30 \mathrm{ps}$ is used to make sure that local equilibration is reached each time after the force is applied, so as to obtain the lowest energy configuration at a given folding position (i.e., the degree of fold), and hence to guarantee that the lowest barrier is obtained, in the unfolding process as well as in the folding process. 


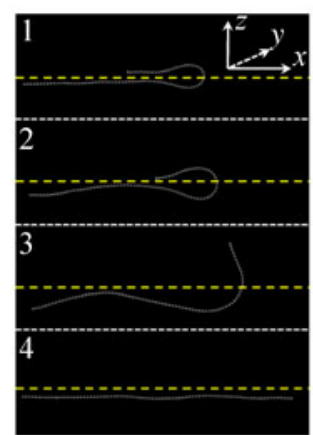

(a)

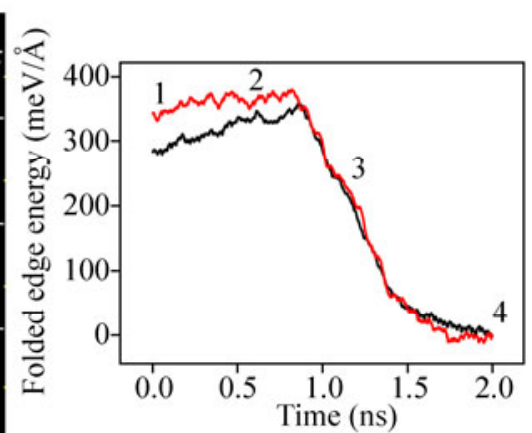

(b)
Figure 2 (a) Structures of SLG during classical molecular dynamics simulation of the unfolding process (from 1 to 4). (b) The folded edge energy as a function of time during the unfolding process (black and red solid lines represent armchair and zigzag edges, respectively). The energy of the flat SLG is set to be zero

The energy per edge atom as a function of time during the unfolding process is shown in Fig. 2(b). Initially, $E_{\mathrm{e}}$ increases due to a decrease in the interlayer bonding vdW energy by virtue of the reduction in the area of the overlapping portion, and subsequently decreases due to the decrease in bending energy associated with unfolding the folding portion. Finally, the SLG sheet becomes flat (Fig. 2(a3)). The energy barriers for edge folding of the SLG (in reference to the flat edge, Fig. 2(a4)) are approximately $360 \mathrm{meV} / \AA$ (or $780 \mathrm{meV} / \mathrm{atom}$ ) and $380 \mathrm{meV} / \AA$ (or $830 \mathrm{meV} /$ atom) for armchair and zigzag edges, respectively; these values are about 30 times larger than the thermal energy per atom at room temperature (RT, $26 \mathrm{meV} /$ atom).

The folded-edge structure can also be obtained by bonding of the top and bottom edges of the DLG (Fig. 3). In this case, both edges undergo a much smaller bending to form the folded edge than in the SLG folding process, and hence the energy barrier is expected to be lower. To obtain the energy barrier, a DLG with open edges is first relaxed for 30 ps (Fig. 3(a1)); periodic forces of opposite directions are then applied vertically on the edge atoms along the $z$-direction to push them toward each other (Fig. 3(a2)) to form the closed-edge structure (Fig. 3(a3)). The change in potential energy is calculated and shown in Fig. 3(b). The energy barriers for DLG to form the closed edge structures are about $55 \mathrm{meV} / \AA$ (or $120 \mathrm{meV} /$ atom) for both armchair and zigzag edges.

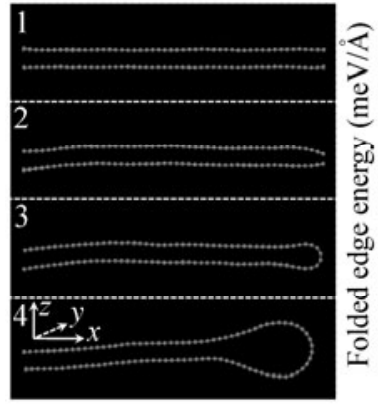

(a)

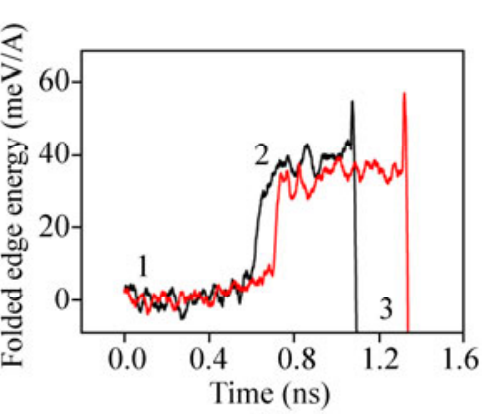

(b)
Figure 3 (a) Structures of DLG during classical molecular dynamics simulation of the folding process (from 1 to 4). (b) The folded edge energy as a function of time during the folding process (black and red solid lines represent armchair and zigzag edges, respectively). The energy of the flat DLG is set to be zero

Given the energy barrier, according transition-state theory, the possibility of edge folding $P$ follows

$$
P \approx v \mathrm{e}^{-E_{\mathrm{b}} / k_{\mathrm{B}} T}
$$

where $E_{\mathrm{b}}, k_{\mathrm{B}}, v$ and $T$ are the energy barrier, Boltzmann constant, attempt frequency and temperature, respectively. $v$ is related to the vibrational frequency of edge atoms. Since the edge atoms have to vibrate in a "collective" manner in order to fold, the attempt frequency is expected to be much smaller than the single atom vibration frequency (the typical order of the atomic vibration frequency is $\sim 10^{13} \mathrm{~s}^{-1}$ ). At RT, the $\mathrm{e}^{-E_{\mathrm{b}} / k_{\mathrm{B}} T}$ factor is about $\sim 10^{-14}$ for the folding of SLG, and $\sim 10^{-2}$ for the folding of DLG. Consequently, at RT the probability of thermal edge folding of both SLG and DLG is very small. But at high temperatures, thermal folding of DLG is feasible. This is consistent with the experiments of Huang et al. who observed spontaneous edge folding of DLG at $k T \approx 200 \mathrm{meV}$ [10] and quantum molecular dynamics results at $k T \approx$ $90 \mathrm{meV}$ [13].

Next, we explore the chemisorption-assisted edge folding process (chemical folding) since, as discussed above, atomic and molecular adsorbates are probably involved in the experimental folding process. It has been shown that chemisorption of $\mathrm{H}$ atoms leads to a change in the hybridization of the carbon atom where $\mathrm{H}$ is adsorbed from $\mathrm{sp}^{2}$ to $\mathrm{sp}^{3}[14]$. If $\mathrm{H}$ atoms are adsorbed only on one side of graphene (or on both 
sides with different coverage), then they will introduce a tensile stress in the graphene sheet providing a driving force for rolling up the edge of graphene [14]. Assuming one-side adsorption, the stress increases with $\mathrm{H}$ coverage and goes up to $800 \mathrm{meV} / \AA^{2}$ [14] at the experimental upper limit of $\mathrm{H}$ coverage on a graphite surface [17]. Recent experiments have also achieved scrolling up of flat graphene by atom/molecule adsorption $[18,19]$. To test the feasibility of chemical folding of graphene edges, for simplicity, we use $\mathrm{H}$ atoms adsorbed on one side of graphene in order to explore the effect of adsorbates. We first derived the energy barrier as a function of $\mathrm{H}$ coverage (Fig. 4), from the energy curves like those in Figs. 2 and 3 for the process without $\mathrm{H}$. The $E_{\mathrm{e}}-l$ curve for the SLG edge folding at $5 \% \mathrm{H}$ coverage is shown in Fig. S-1 as an example. Our results show that the energy barrier of folding decreases significantly as $\mathrm{H}$ coverage increases. At $\sim 14 \% \mathrm{H}$ coverage, the energy barrier vanishes, which means that the edge of SLG can fold spontaneously at this $\mathrm{H}$ coverage or higher.

Furthermore, we performed MD simulations to directly observe the chemical folding process. First, a sheet of SLG without adsorbate is relaxed for 30 ps. Then hydrogen atoms at $\sim 50 \%$ coverage $^{1}$ are absorbed on the bottom side of suspended graphene with a random distribution. Driven by the stress induced by

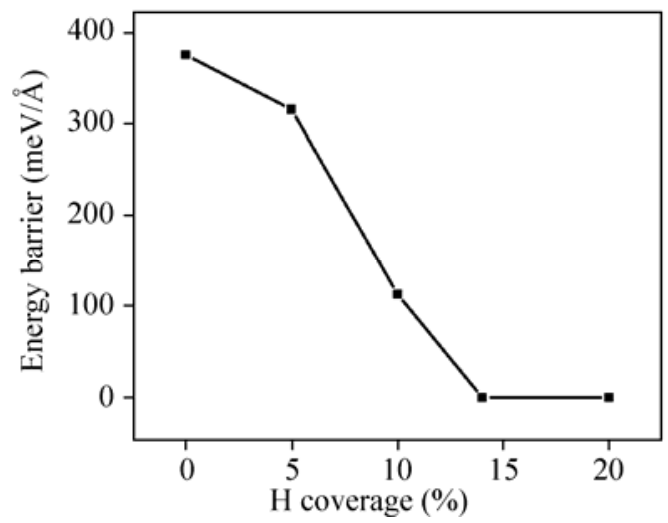

Figure 4 The energy barrier for SLG edge folding as a function of $\mathrm{H}$ coverage hydrogen adsorption, the edge begins to bend up and finally forms the folded structure (a movie showing this process is available in the Electronic Supplementary Material (ESM)).

Similarly, a DLG is relaxed for 30 ps, and then $30 \%$ $\mathrm{H}$ coverage $\mathrm{e}^{1}$ is introduced at the near-edge region of both layers. The edge of the upper layer bends down while the edge of the bottom layer bends up and they meet and bond together to form a closed structure. Finally, the curved portion expands to release strain energy until $\partial E_{\text {strain }} / \partial L=-\partial E_{\mathrm{vdW}} / \partial L$, where $E_{\text {strain }}$ and $E_{\mathrm{vdW}}$ are the bending strain energy and the interfacial vdW bonding energy, respectively (a movie showing this process is available in the ESM). These results demonstrate that the edge of graphene may fold spontaneously with sufficiently high one-side (or imbalanced two-side ${ }^{2}$ ) hydrogen adsorption at room temperature, indicating a spontaneous chemical folding process. It is interesting to see that chemical adsorption of $\mathrm{H}$ promotes the edge folding of DLG, while in contrast, vacancies and impurities inhibit the edge folding of DLG [13].

Although we have only studied $\mathrm{H}$ adsorption here, other adsorbates may have the same effect. One basic condition is for the adsorbates to induce a tensile surface stress and fold the graphene by converting the $\mathrm{C}$ bonding configuration from $\mathrm{sp}^{2}$ to $\mathrm{sp}^{3}$. One example is $\mathrm{F}$, which has previously been shown to fold graphene nanoribbons into nanotubes [14]. We also note that if graphene is on a substrate other than graphite, our approach will work best with a very weak interaction between graphene and the substrate, such as the van der Waals type of interaction found for graphite itself.

In conclusion, we have derived for the first time the kinetic energy barriers for bending of SLG and DLG edges to form a closed edge structure. Based on the energy barriers, we conclude that thermal edge folding of SLG is generally not feasible, while thermal edge folding of DLG is feasible at high temperature but not at room temperature. However, edge folding barriers can be greatly reduced by imbalanced chemisorption

\footnotetext{
${ }^{1}$ To ease the simulation and save time, here we choose a high one-sided $\mathrm{H}$ coverage to accelerate the edge folding process.

${ }^{2}$ It is interesting to note that when H is adsorbed on both sides of a SLG, the coverage on the two sides can initially be different. Then the SLG will start to fold towards the side with less H. As a result, the outer side with more $\mathrm{H}$ will be exposed and be more accessible for additional $\mathrm{H}$ adsorption, while the inner side with less $\mathrm{H}$ will be "shielded" from $\mathrm{H}$ adsorption. This will enhance the imbalance of $\mathrm{H}$ coverage on the two sides, further assisting the folding process.
} 
of atoms or molecules (as demonstrated for $\mathrm{H}$ ) on the two sides of graphene, which may even lead to spontaneous edge folding of both SLG and DLG at room temperature. Our findings indicate that the formation of suspended graphene with folded edges as observed in some experiments at room temperature [7-9] may involve assistance by adsorbates.

\section{Acknowledgements}

This work was supported by DOE (Nos. DE-FG0203ER46027, DE-SC0001061); NSF-DMR (No. 0909212). The atomic structures in all figures and movies are visualized using the Jmol, an open-source Java viewer for chemical structures in 3D: http://www.jmol.org/.

Electronic Supplementary Material: Movies of molecular dynamics simulations showing folding/unfolding processes of both SLG and DLG are available in the online version of this article at http://dx.doi.org/ 10.1007/s12274-011-0175-0.

\section{References}

[1] Yan, Q.; Huang, B.; Yu, J.; Zheng, F.; Zang, J.; Wu, J.; Gu, B.; Liu, F.; Duan, W. Intrinsic current-voltage characteristics of graphene nanoribbon transistors and effect of edge doping. Nano Lett. 2007, 7, 1469-1473.

[2] Fasolino, A.; Los, J. H.; Katsnelson, M. I. Intrinsic ripples in graphene. Nat. Mater. 2007, 6, 858-861.

[3] Huang, B.; Liu, F.; Wu, J.; Gu, B.; Duan, W. Suppression of spin polarization in graphene nanoribbons by edge defects and impurities. Phys. Rev. B 2008, 77, 153411.

[4] Shenoy, V. B.; Reddy, C. D.; Ramasubramaniam, A.; Zhang, Y. W. Edge-stress-induced warping of graphene sheets and nanoribbons. Phys. Rev. Lett. 2008, 101, 245501.

[5] Bets, K. V.; Yokobson, B. I. Spontaneous twist and intrinsic instabilities of pristine graphene nanoribbons. Nano Res. 2009, 2, 161-166.

[6] Huang, B.; Liu, M.; Su, N.; Wu, J.; Duan, W.; Gu, B.; Liu, F. Quantum manifestations of graphene edge stress and edge instability: A first-principles study. Phys. Rev. Lett. 2009, 102, 166404.
[7] Meyer, J. C.; Geim, A. K.; Katsnelson, M. I.; Novoselov, K. S.; Booth, T. J.; Roth, S. The structure of suspended graphene sheets. Nature 2007, 446, 60-63.

[8] Gass, M. H.; Bangert, U.; Bleloch, A. L.; Wang, P.; Nair, R. R. Free-standing graphene at atomic resolution. Nat. Nanotechnol. 2008, 3, 676-681.

[9] Liu, Z.; Suenage, K.; Harris, P. J. F.; Iijima, S. Open and closed edges of graphene layers. Phys. Rev. Lett. 2009, 102, 015501.

[10] Huang, J. Y.; Ding, F.; Yakobson, B. I.; Lu, P.; Qi, L.; Li, J. In situ observation of graphene sublimation and multi-layer edge reconstructions. P. Natl. Acad. Sci. U.S.A. 2009, 106, 10103-10108.

[11] Yu, W. J.; Chae, S. H.; Perello, D.; Lee, S. Y.; Han, G. H.; Yun, M.; Lee, Y. H. Synthesis of edge-closed graphene ribbons with enhanced conductivity. ACS Nano 2010, 4, 5480-5486.

[12] Cranford, S.; Sen, D.; Buehler, M. J. Meso-origami: Folding multilayer graphene sheets. Appl. Phys. Lett. 2009, 95, 123121.

[13] Cruz-Silva, E.; Botello-Méndez, A. R.; Barnett, Z. M.; Jia, X.; Dresselhaus, M. S.; Terrones, H.; Terrones, M.; Sumpter, B. G.; Meunier, V. Controlling edge morphology in graphene layers using electron irradiation: From sharp atomic edges to coalesced layers forming loops. Phys. Rev. Lett. 2010, 105, 045501.

[14] Yu, D.; Liu, F. Synthesis of carbon nanotubes by rolling up patterned graphene nanoribbons using selective atomic adsorption. Nano Lett. 2007, 7, 3046-3050.

[15] Brenner, D. W.; Shenderova, O. A.; Harrison, J. A.; Stuart, S. J.; Ni, B.; Sinnott, S. B. A second-generation reactive empirical bond order (REBO) potential energy expression for hydrocarbons. J. Phys.-Condens. Mat. 2002, 14, 783-802.

[16] Girifalco, L. A.; Lad, R. A. Energy of cohesion, compressibility, and the potential energy functions of the graphite system. $J$. Chem. Phys. 1956, 25, 693-697.

[17] Zecho, T.; Guttler, A.; Sha, X. W.; Jackson, B.; Kuppers, J. Adsorption of hydrogen and deuterium atoms on the (0001) graphite surface. J. Chem. Phys. 2002, 117, 8486-8492.

[18] Xie, X.; Ju, L.; Feng, X.; Sun, Y.; Zhou, R.; Liu, K.; Fan, S.; Li, Q.; Jiang, K. Controlled fabrication of high-quality carbon nanoscrolls from monolayer graphene. Nano Lett. 2009, 9 , 2565-2570.

[19] Sidorov, A.; Mudd, D.; Sumanasekera, G.; Ouseph, P. J.; Jayanthi, C. S.; Wu, S. Y. Electrostatic deposition of graphene in a gaseous environment: A deterministic route for synthesizing rolled graphenes. Nanotechnology 2009, 20, 055611. 